\title{
Panorámica general de los grupos de investigación en Antropología Social en el Estado español
}

\author{
Research groups in Social Anthropology in Spain: \\ an overview
}

\author{
Jesús SANZ ABAD \\ Departamento de Antropología Social. Universidad Complutense de Madrid \\ jesusanz@cps.ucm.es
}

Recibido: 31 de mayo de 2011

Aceptado: 22 de junio de 2011

\section{Resumen}

A modo de introducción y presentación de este volumen monográfico, se repasa la evolución de la Antropología Social en el Estado español y de sus temáticas predominantes, trazando una panorámica general sobre la situación de la investigación antropológica desarrollada en la actualidad a partir del análisis de los grupos participantes en este monográfico y de otros equipos existentes. En la segunda parte del texto, se hace un breve comentario sobre las características temáticas y teórico-metodológicas de los artículos presentados, que nos invita a reflexionar acerca de las transformaciones y novedades más relevantes que se han ido produciendo en la disciplina. Asimismo se resalta el papel central de la etnografía como principal seña de identidad de la Antropología Social en el nuevo contexto analítico de la globalización.

Palabras clave: Antropología Social, grupos de investigación en el Estado español, estado de la cuestión, etnografía, temas de estudio.

\begin{abstract}
In this introduction and presentation of this monography, a review is done on the evolution of Social Anthropology in Spain, as well as the evolution of the topics that have prevailed under the discipline. Insofar as there is an analysis of the groups represented or not in this volume, there is an overview on the current status of Anthropological research Spain. In the second part of the text, the reader will find a brief comment on the theoretical and methodological characteristics collected in the texts. In light of the texts there is a reflection on the relevant changes and developments that have taken place in the discipline. It also highlights the central role of ethnography as the main hallmark of Anthropology in the new context of globalization analysis.
\end{abstract}

Keywords: SocialAnthropology, research groupsinSpain, state oftheart, ethnography, research topics.

Referencia normalizada: Sanz Abad, J. (2011). Panorámica general de los grupos de investigación en Antropología Social en el Estado español. Revista de Antropología Social, 20, 9-29. 
SUMARIO: 1. Breve recorrido por la evolución de la Antropología Social en el Estado español. 2. Panorámica general de los grupos de investigación en Antropología Social en el ámbito estatal. 3. Presentación del monográfico: temas clásicos y nuevos dominios en el estudio de las sociedades contemporáneas. 4. Últimos comentarios. 5. Referencias bibliográficas.

\section{Breve recorrido por la evolución de la Atropología Social en el Estado español}

En 1999 un estudio coordinado por Joan Prat compiló la investigación antropológica efectuada en el Estado español durante los últimos 40 años, analizando su evolución desde 1954 a 1998 y reuniendo más de 8000 referencias bibliográficas. Además de realizarse una extraordinaria recopilación bibliográfica y un buen estado de la cuestión, se examinaba la evolución temática producida. Así, se señalaban diversos acontecimientos destacables que habían marcado el devenir de esta disciplina $^{1}$ a nivel estatal, identificando distintas etapas en su proceso de consolidación.

En primer lugar, hay consenso en vincular el nacimiento de la antropología moderna en el Estado español con la publicación del trabajo de Pitt Rivers "The people of the Sierra" en 1954. Esta aportación supone el inicio de otras promovidas por diferentes antropólogos procedentes de Oxford - donde Evans Pritchard impartía docencia en aquellos años-, entre las que sobresalen las llevadas a cabo por Carmelo Lisón. De la misma forma, en los pasados años sesenta, desde varias universidades norteamericanas se acometen diversos trabajos y tesis doctorales.

En términos generales, estas iniciativas se correspondían con los planteamientos estructural-funcionalistas hegemónicos en la época, y buscaban la realización de estudios de comunidad durante un periodo de tiempo prolongado a fin de captar, analizar e interpretar de forma global la cultura de los lugareños. A su vez, buena parte de estos trabajos estaban realizados en comunidades rurales y mal comunicadas, por lo que era fácil representarlas como estructuras aisladas y autosuficientes.

Desde los últimos años setenta, una segunda línea de investigación se centra en la búsqueda de colectivos o comunidades que eran representados como formas próximas de alteridad. En este contexto se inscriben diversas actuaciones sobre algunos sujetos estigmatizados, considerados "grupos marginados": gitanos, vaqueiros de Alzada, pasiegos, agotes, maragatos o chuetas. Paralelamente, en esa búsqueda de nuevas formas de alteridad, se dirige el foco hacia el campesinado y las transformaciones operadas en el mundo rural. Del mismo modo, estas construcciones enfatizarán el papel del parentesco en la vida social tradicional, al igual que la mayoría de tratados de comunidad citados antes.

Una tercera etapa la definen la llamada España de las autonomías y el proceso de descentralización universitario, originado desde fines de los pasados años setenta y principios de los ochenta. En este momento comienza la progresiva institucionalización profesional de la disciplina con la creación de los primeros departamentos de Antropología. Asimismo en estos años se despliega en el plano teórico una crítica a los postulados funcionalistas y estructural-funcionalistas de trabajos previos. Se

${ }^{1}$ Para un abordaje más profundo de este asunto, me remito a la excelente recopilación coordinada por Joan Prat (1999). De ella extraigo los datos aquí presentados. 
genera también un interés especial por la identidad y algunas temáticas relacionadas con ella - la cultura popular, el folklore, o la fiesta como elemento configurador de cohesión social e identitario, entre otros dominios-, lo que no resulta llamativo en un contexto político, marcado por la aprobación de los diferentes estatutos de autonomía y el incipiente proceso descentralizador del Estado.

Simultáneamente y ligado al acento en la identidad, algunos investigadores desplazarán su mirada del mundo rural al urbano y, singularmente, a sus periferias. Con ello, desde mediados de los últimos años ochenta, se abordan cuestiones específicas sobre inserción social y laboral, procesos de marginación étnica, "subculturas de la pobreza", nuevas inmigraciones, o diferentes colectivos estigmatizados por su condición de marginación: drogadictos, homosexuales y otros.

Finalmente, la proliferación de nuevas temáticas de trabajo, advertidas desde los pasados años noventa, hace difícil señalar el predominio concreto de alguna de ellas, una tendencia que, como veremos, se ha multiplicado hasta la actualidad. Según señala Prat en su texto, desde los últimos años noventa, la Antropología Social ha unido, a los dominios más clásicos, otros asuntos tan dispares como el género, la salud, la medicina, la denominada antropología de las edades, la alimentación, el deporte, la pesca, el turismo, la antropología industrial y de la empresa, la educación o el patrimonio. Ello dibuja sin duda un panorama rico y heterogéneo.

\section{Panorámica general de los grupos de investigación en Antropología Social en el ámbito estatal}

Partiendo de esa revisión de Joan Prat, este número monográfico, dedicado por la Revista de Antropología Social a los grupos de investigación existentes en el marco estatal y a sus análisis, nos brinda una buena ocasión para hacer un balance del panorama de trabajo que define a la disciplina en dicho ámbito.

Obviamente el contenido del monográfico lo determinan las limitaciones impuestas por la propia publicación, por lo que se seleccionó a los participaciones siguiendo criterios ajustados al contexto específico de la Revista. Se ha tratado de primar la incorporación de equipos que, estando académicamente consolidados, cuentan ya con una dilatada trayectoria de estudio. En la medida de lo factible, se ha procurado ofrecer una muestra de la diversidad temática desarrollada, recurriendo, en ciertos casos, a categoríass más operativas que académicas, como potenciar la cooperación de colectivos integrados mayoritariamente por antropólogos o invitar, en asuntos puntuales, a aquellos que todavía no habían colaborado en alguna publicación reciente de la Revista de Antropología Social. Lógicamente, esto último ha supuesto la aceptación de los convocados a comprometerse en esta edición, intervención que reconocemos y agradecemos por su gran valor.

El resultado ha sido un monográfico que recoge iniciativas de miembros de los siguientes equipos: Grupo de Estudios sobre Reciprocidad (GER); Grupo de Estudios sobre Migración, Etnicidad y Ciudadania; Antropoloxia; Grupo Transformaciones Sociales y Culturales en Andalucía y América; Grupo Investigación Social y Acción Participativa (GISAP); Grupo Cultura, Ecología y Desarrollo de Pequeños Territorios; Grupo de Investigaciones en Antropología (GIA); Grupo de 
Investigación en Antropología Fundamental y Orientada (GRAFO); Laboratorio de Estudios Interculturales (LdEI); Antropología e Historia de la Construcción de Identidades Sociales y Políticas (AHICISP); y Antropología de las Políticas Sociales y Culturales (APSYC).

Al analizar estas aportaciones, resulta llamativa, en primer lugar, su distribución territorial profundamente desigual, que parece estar relacionada de modo directo con el grado de institucionalización y la existencia o no de estudios reglados de Antropología Social en diferentes áreas. No es casual que Madrid, Catalunya y Andalucía constituyan los lugares donde la disciplina tiene una mayor presencia, al ser los únicos en que se oferta esta docencia en varias universidades.

En este sentido, hay que subrayar que, dentro del proceso de reestructuración generado por la sustitución de la antigua Licenciatura por un grado de Antropología Social y Cultural de nueva creación, los centros que hasta el momento han ofertado estudios de grado son: la Universidad Complutense de Madrid, Universidad Autónoma de Madrid, Universidad Nacional de Educación a Distancia, Universitat Autónoma de Barcelona, Universitat de Barcelona, Universidad de Sevilla, Universidad de Granada y Universidad del País Vasco. Asimismo, en la Universitat Rovira i Virgili de Tarragona, se está examinando la implantación de un grado de Antropología conjunto con otra especialidad. De igual modo, son varias las universidades que ofrecen diferentes estudios de Máster de Antropología más o menos especializados en alguna orientación concreta ${ }^{2}$.

Desde el punto de vista territorial, se observa también un nexo directo entre el desigual arraigo de la Antropología y la ubicación de los equipos de trabajo considerados. Salvo en el caso del grupo Antropoloxia, que pertenece a la Universidad de Santiago de Compostela, el resto de los participantes en este volumen se localiza en alguna de las tres áreas ya citadas, y en todos los casos están vinculados a universidades que ofrecen programas oficiales de Antropología Social. Se muestra así una relación sin apenas intermediación entre el grado de institucionalización académica y la existencia o no de grupos de investigación en los diferentes territorios, que alude, a su vez, al paralelismo disciplinar de la oferta universitaria con la investigación.

${ }^{2}$ Entre los másteres organizados por diferentes departamentos de Antropología Social, hay que citar los siguientes: "Estudios Avanzados de Antropología Social", de la Universidad Complutense de Madrid; "Antropología de Orientación Pública", de la Universidad Autónoma de Madrid; "Migraciones", "Antropología Urbana” y "Antropología Médica y Salud Internacional”, ofrecidos los tres por la Universitat Rovira i Virgili; "Antropología y Etnografía” y "Gestión del Patrimonio", ambos de la Universitat de Barcelona; "Investigación Etnográfica", de la Universidad Autónoma de Barcelona; "Interculturalidad, Patrimonio y Desarrollo", de la Universidad de Sevilla; y "Nuevas Tendencias en Antropología: Escenarios de Riesgo y Alternativas de Postdesarrollo", de la Universidad Miguel Hernández. Por último, la Universidad del País Vasco también dispone de un máster interdisciplinar en "Estudios Feministas y de Género" — especialidad en Antropología, Género y Feminismos-, que es coordinado desde la sección departamental de Antropología Social. 
Tres grandes tipos clasificarían a los colectivos que vertebran este monográfico.

- El primero agruparía a los más generalistas y con una marcada orientación transversal. En él se puede englobar, al menos, al "Grup d'Antropología Social" de la Universitat Rovira i Virgili y, en menor medida, al equipo "Transformaciones Socioculturales en Andalucía y América Latina".

El "Grup d'Antropología Social" lo forma el profesorado del Departamento de Antropología, Filosofía y Trabajo Social de la URV, aunque cuenta también con algunos miembros externos a la institución. Es amplio, disponiendo de 40 investigadores, interdisciplinario y diverso, si bien su eje central, como indica su nombre, se configura alrededor de la disciplina antropológica ${ }^{3}$.

En los últimos años, sus temas versan, entre otras, sobre procesos de metropolización y reordenamiento territorial; multiculturalismo; prácticas ligadas a procesos migratorios; y nuevas prácticas de organización social y familiar, así como en bienestar, salud y alimentación.

Los integrantes de "Transformaciones Socioculturales en Andalucía y Ameríca Latina”, de la Universidad de Sevilla ${ }^{4}$, oscilan entre la orientación generalista y la especialización en un tema concreto. Entre ellos, hay varios investigadores doctores, pertenecientes a diversas universidades. Desde una dimensión trasnacional, perspectivista, comparativa y crítica, se ocupan de asuntos articulados con procesos identitarios, políticos y religiosos contemporáneos, sobresaliendo los entroncados con movimientos sociales, religión e identidad. Así lo expresan sus elaboraciones sobre los movimientos socio-religiosos, étnicos y políticos en América Central, Sureste de México y Andalucía occidental, la conexión entre movimientos sociales e identidades de género, o la construcción política y los procesos de etnogénesis del protestantismo gitano.

- Un segundo tipo se define por una línea de actuación más específica. Aquí encuadraríamos a los componentes de "Antropoloxia", del "Grupo de Estudios sobre Reciprocidad", de "Antropología e Historia de la Construcción de las Identidades Sociales y Políticas", y de "Antropología de las Políticas Sociales y Culturales".

"Antropoloxia" lo forman varios miembros del Área de Antropología Social de la Universidad de Santiago de Compostela ${ }^{5}$. Estudia preferentemente el patrimonio cultural, la antropología del turismo y el desarrollo local. Durante los últimos años

\footnotetext{
${ }^{3}$ Además de los colectivos de Barcelona, reseñados más adelante, y de este grupo de la URV, está "Mediaciones: Estudio sociocultural de Internet, las tecnologías y los nuevos medios", de la Universitat Oberta de Catalunya (UOC). Por otro lado, antropólogos de la Universitat de Lleida participan en el "Grupo Interdisciplinario de Estudios sobre Desarrollo y Multiculturalidad" y en el "Grupo de Estudios de las Sociedades Africanas". Finalmente, antropólogos de la Universitat de Girona son miembros del interdisciplinar "Grupo de Estudios Sociales de la Pesca Marítima".

${ }^{4}$ Junto a los participantes en este monográfico, de la Universidad de Sevilla, otros colegas de su mismo centro componen el "Grupo para el Estudio de las Identidades Socioculturales en Andalucía (Geisa)", el "Grupo de Investigación y Estudios sobre la Religión en Andalucía”, el "Grupo de Etnografía y Mediaciones de Comunicación y Desarrollo (Etnomedia-CD)" y el "Grupo sobre Patrimonio Etnológico, Recursos Socio-Económicos y Simbolismo".

${ }^{5}$ En Galicia también hay que citar la participación de algunos antropólogos de la Universidad de A Coruña en el "Grupo de Investigación Social Aplicada". Además, diversos antropólogos investigan
} 
ha llevado a cabo distintos proyectos en Galicia, enfocados a los usos de la cultura y del patrimonio cultural como factores de crecimiento, propiciados por el turismo, y a sus efectos sobre las economías e identidades locales.

Por su parte, el "Grupo de Estudios sobre Reciprocidad" pertenece al Departamento de Antropología Cultural e Historia de América y África de la Universitat de Barcelona ${ }^{6}$. Se origina en 1998 con un proyecto financiado sobre "La reciprocidad como recurso humano: el uso de relaciones informales abiertas en los ámbitos de la economía y la política". Desde entonces, se ha centrado en aspectos ligados a la regulación no contractual de la circulación de recursos económicos y, muy especialmente, a la reciprocidad, entendiéndola como aquellas relaciones sociales de larga duración que sustentan flujos de transferencias sobre una moral de obligación mutua diferente a la del contrato. Bajo estos presupuestos y una apuesta metodológica basada en el método etnográfico y el análisis comparativo, ha tratado los siguientes asuntos: acceso a la vivienda y recursos sociales y alternativos empleados en contextos mercantiles hegemónicos; cuidado de la gente mayor y vías de aprovisionamiento del mercado; parentesco, voluntariado y Estado; ordenación jurídica y políticas públicas de regulación en contextos de responsabilidad no instituida legalmente; o los efectos de la desindustrialización en el trabajo y las formas alternativas e informales de acceso a necesidades básicas.

El "Grupo de Antropología e Historia de la Construcción de las Identidades Sociales y Políticas (AHCISP)", adscrito a Universitat Autónoma de Barcelona, también se centra en una línea temática específica: etnografía y antropología histórica desde una perspectiva crítica del dualismo cartesiano que opone naturaleza y cultura y condiciona la construcción de las identidades y exclusiones socio-políticas en distintos contextos históricos y geográficos. De modo más concreto, aborda, por un lado, la creación de identidades y exclusiones en la formación de los Estados nacionales, la nacionalidad y los derechos de ciudadanía; la emergencia de procesos identitarios socio-políticos; y la etnogénesis de nuevas identidades. Por otra parte, incide en los significados simbólicos, supuestos ontológicos e intersecciones concernientes, entre otras, a categorías como naturaleza y cultura, raza y género, racismo y etnicidad, individuo y sociedad, o mestizaje e hibridación. Unido a ello, le interesa asimismo el modo en que las fronteras de estas categorías son transgredidas.

interdisciplinarmente el patrimonio cultural en el Laboratorio de Patrimonio, adscrito al CSIC y cuya sede se encuentra en Santiago de Compostela.

${ }^{6}$ Igualmente, en la Universitat de Barcelona, hay que mencionar al "Grup de Recerca Cultures Indígenas i Afroamericanes: Història(es), Identitats i Ciutadania (CINAF)" y al "Grup d'Estudis sobre Familia i Parentiu". Éste último atiende sobre todo la continuidad y los cambios en modelos familiares y relaciones de parentesco — reproducción y transmisión del patrimonio, políticas familiares o papel de la familia en la organización socioeconómica - y las implicaciones socioculturales de las terapias de infertilidad - representaciones sobre el cuerpo, la naturaleza y las relaciones sociales; o procesos de medicalización y mercantilización reproductiva- La Revista de Antropología Social ha publicado en 2009 un artículo de Joan Bestard, "Los hechos de la reproducción asistida: entre el esencialismo biológico y el constructivismo social", que puede servir de referente de una de estas orientaciones más novedosas. A la par, el "Grup de Recerca en Exclusió i Control Social”, de carácter interdisciplinar, incluye miembros del departamento de Antropología Social e Historia de América y África de la UB. 
Finalmente, el "Grupo Antropología de las Políticas Sociales y Culturales (APSYC)", integrado por miembros del Departamento de Antropología Social de la Universidad Complutense de Madrid, analiza las políticas sociales y la intervención en contextos de transformación social bajo un enfoque etnográfico y crítico $^{7}$. Se ha ocupado de las circunstancias socioculturales que rodean los nuevos movimientos reivindicativos sociales y políticos, la relación de los colectivos sociales con las políticas públicas de intervención, y las asimetrías entre las ofertas públicas y su recepción por los ciudadanos. A la vez, ha prestado atención a los procesos de activación de los recursos culturales promovidos desde instituciones políticas formalizadas, o a la construcción diferencial operada en la intervención política en categorías como público y privado, derechos y deberes, o cultura y patrimonio.

- En un tercer tipo estarían los equipos que, de manera más explícita, consideran prioritaria una antropología orientada y aplicada: el "Laboratorio de Estudios Interculturales", "Migraciones, Etnicidad y Ciudadanía", "Cultura, Ecología y Desarrollo en Pequeños Territorios", "GRAFO (Grup de Recerca en Antropología Fonamental i Orientada)", y "Grupo de Investigación Social y Acción Participativa (GISAP)". No obstante, en muchos casos, su labor también está presidida por una línea específica de trabajo, por lo que no es fácil establecer una delimitación clara y agrupar a algunos de ellos en este apartado o en el anterior. Sorprende, por lo demás, que tres de estos colectivos: "Laboratorio de Estudios Interculturales", "Migraciones, Etnicidad y Ciudadanía" y "EMIGRA", estén, de uno u otro modo, tan volcados hacia las migraciones.

El "Laboratorio de Estudios Interculturales (LdEI)", de la Universidad de Granada y perfil interdisciplinar ${ }^{8}$, está conformado por profesores e investigadores especializados en diversas áreas de las ciencias sociales: Antropología, Sociología, Pedagogía, Psicología o Trabajo Social. Se ocupa, entre otros asuntos, de migraciones, etnicidad, interculturalidad, racismo, educación intercultural y procesos de integración y exclusión de la población inmigrante. Combina investigación, formación, docencia, difusión y otras actividades, muchas de ellas de tipo práctico, sobre migración, interculturalidad y multiculturalismo, racismo, xenofobia, conflictos

${ }^{7}$ En la Universidad Complutense de Madrid, no olvidamos tampoco la presencia de antropólogos en equipos interdisciplinares: "EGECO (Empleo, Género y Cohesión Social)” y "ACIS (Investigación y mitocrítica)"; iniciativas especialmente en el campo del patrimonio, de la antropología de la salud, de los derechos humanos y del parentesco; y múltiples proyectos ejecutados desde el departamento de Historia de América II (Antropología de América), así como el trabajo realizado desde el "Grupo de Estudios de Etnología Americana".

${ }^{8}$ De la Universidad de Granada dependen asimismo estos equipos: "AFRICAInEs-Investigación y Estudios Aplicados al Desarrollo", "Antropología Social de la Motricidad”, "Estudios Socioculturales Aplicados", "Género: Historia y Antropología", "Observatorio de Prospectiva Cultural”, "Antropología y Filosofía”, y “Otras: Perspectivas Feministas en la Investigación Social”. Más allá de los colectivos de Sevilla y Granada, citados en este artículo, el panorama en Andalucía se complementa, al menos, con la existencia de algunos otros, pertenecientes a las Universidades de Almería y Jaén. En Almería, registramos al "Laboratorio de Antropología Social y Cultural" y a "Nuevas Proyecciones para el Patrimonio de la Cultura Oral y Popular: Educación, Museos y Turismo" que oscila entre la Antropología y la Literatura. En Jaén cabe mencionar a "Una Mirada Antropológico-social desde Andalucía” y "Antropología e Historia de la Cultura". 
étnicos y nacionalismo, formas de discriminación y exclusión de género. De él depende un Centro de Documentación en Interculturalidad.

"Migraciones, Etnicidad y Ciudadanía", de la Universidad Autónoma de Madrid, dispone de 16 integrantes que reflexionan sobre migraciones internacionales, modelo de ciudadanía y procesos identitarios desde una perspectiva transnacional, de género, generación y clase ${ }^{9}$ y bajo una dimensión pública, aplicada y comprometida con la construcción de relaciones sociales equitativas e interculturales. Sus últimas experiencias versan acerca de las representaciones del contexto migratorio a nivel mediático y de propuestas teórico-prácticas relativas a la conflictividad y migración en contextos locales.

"Cultura, Ecología y Desarrollo de Pequeños Territorios", de la Universidad de Sevilla, fundado en 2000 y con 15 componentes, opera también con una temática concreta y una clara vocación por una antropología aplicada y orientada a la resolución de problemas sociales. Colabora con agencias públicas, de actuación social y entidades territoriales en la planificación y gestión de programas de desarrollo. Se ha especializado en el análisis local de variables sociales y culturales, recursos propios, agroecosistemas tradicionales, articulaciones entre biodiversidad y cultura, y en su contribución a los procesos de desarrollo territorial.Finalmente, hallamos al "Grup de Recerca en Antropología Fonamental i Orientada (GRAFO)" y al "Gupo de Investigación Social y Acción Participativa (GISAP)", sin una línea de investigación temática claramente definida pero sí con una clara vocación orientada y aplicada.

El primero de ambos, adscrito al Departamento de Antropología Social y Cultural de la Universitat Autònoma de Barcelona ${ }^{10}$, intenta consolidar las bases teóricas de la Antropología y sus estudios mediante la reflexión de sus supuestos ontológicos, epistemológicos y metodológicos y buscando la conceptualización y el desarrollo de procedimientos instrumentales que fundamenten la adecuación entre los diseños de investigación y las técnicas empleadas. Muchas de las actuaciones del "GRAFO", sobre todo en el ámbito de la salud y las instituciones educativas, procuran la transformación de las condiciones socioculturales con una concepción de

${ }^{9}$ En la Universidad Autónoma de Madrid diversos antropólogos cooperan con el "Taller de Estudios Internacionales Mediterráneos" y el "Instituto Universitario de Investigación sobre Migraciones, Etnicidad y Desarrollo Social (IMEDES)". En Madrid, también hay que señalar a "Familia y Parentesco en el Siglo XXI" y "Cultura Urbana" de la UNED. Por su parte, en la sede madrileña del Consejo Superior de Investigaciones Científicas (CSIC), se encuadran el "Grupo de Investigación Antropológica sobre Patrimonio y Culturas Populares (GIAP)" y el "Grupo de Investigación de Antropología Comparada de España y América".

${ }^{10}$ En del departamento de Antropología Social de la Universitat Autónoma de Barcelona (UAB), tampoco debe obviarse a "EMIGRA", que trabaja sobre infancia y educación en contextos migratorios; "EGOLAB", que aborda las redes sociales; "Grupo de Estudio Transcultural del Parentesco (GETP)"; "Grup de Investigación en Etnografía de los Procesos Educativos y de Antropología Aplicada (MOSSA)"; "Grupo de Relaciones Interculturales y Marginación Social (GRIM)"; y "Grupo Interdisciplinar Prevención Estudio de las Prácticas Tradicionales Perjudiciales (IPE-PTP)". Desde este departamento se coopera simultáneamente con "Grupo Interdisciplinar de Investigación Histórica, Ambiental y Cultural” y con “AFIN (Adopciones, Familias, Infancias)”. 
la Antropología aplicada - objeto de una importante teorización - cuyo horizonte es la investigación-acción desde una posición epistemológica crítica. Igualmente, se presta gran atención a las redes sociales.

Por su lado, el "Gupo de Investigación Social y Acción Participativa (GISAP)", de la Universidad Pablo de Olavide de Sevilla, prioriza los estudios aplicados y de intervención a través de metodologías participativas, asesorías y acciones formativas. Trata sobre medio ambiente, patrimonio natural y desarrollo sostenible; política local, gobernanza, participación ciudadana, cultura política y planificación estratégica participativa; culturas del trabajo y de la empresa, organización empresarial y relaciones sociolaborales; ordenación del territorio, urbanismo y procesos de transformación de la ciudad; e intervención y servicios sociales.

En resumen, el balance general que diseñan los equipos de investigación resalta una amplísima variedad temática, que no cesa de multiplicarse con la aparición de nuevas líneas de trabajo, profundizando, con ello, la tendencia ya mostrada por Prat en su estudio de los pasados años 90, por mucho que la migración predomine en la actualidad sobre otros asuntos, según veremos más adelante.

Esa característica se suma a la ya observada relación directa entre el grado de institucionalización académica y la presencia o no de grupos de investigación en los diferentes territorios y su desigual distribución territorial.

También es significativa la escasez de colectivos con carácter interdisciplinar y que cuenten con miembros procedentes de otras disciplinas, a excepción del "Laboratorio de Estudios Interculturales" y de "Antropología e Historia de la Construcción de las Identidades Sociales y Políticas (AHCISP)".

Igualmente, se constata que la mayoría de los integrantes de los equipos están adscritos al mismo departamento y/o a la misma universidad, siendo infrecuente la cooperación interdepartamental e interuniversitaria. Este rasgo, unido a la escasez de redes de investigadores asociados por intereses temáticos, muestra un panorama disciplinar un tanto atomizado y articulado en torno a los diferentes departamentos de Antropología, donde el trabajo en red es más excepción que norma ${ }^{11}$.

A la par, se advierte en bastantes grupos un esfuerzo creciente por la realización de una Antropología, que esté orientada a la intervención y sea capaz de aplicar y poner en práctica los conocimientos adquiridos mediante la investigación.

Sin embargo, somos conscientes de que este panorama va mucho más allá de lo mostrado por los participantes en el presente monográfico y de la información ofrecida en las diferentes notas al pie, relativas a las universidades de Madrid, Cataluña, Andalucía y Galicia. En otros territorios del Estado como la Comunidad

${ }^{11}$ Matiza esta afirmación la excepción que supone, por ejemplo, la Red de Antropología Médica que, aun cuando no realiza propiamente investigaciones, tiene una actividad frecuente y organiza anualmente un coloquio. A su vez, el "Grup d'Estudis sobre Familia i Parentiu", de la UB, y el "Grup de Estudi Transcultural sobre la Procreació", de la UAB, participan en la Xarxa Interdisciplinària d'Estudis sobre la Familia junto a otros colectivos especializados en esta temática. 
Valenciana ${ }^{12}$, Canarias ${ }^{13}$, Navarra ${ }^{14}$ o el País Vasco ${ }^{15}$ existe una notable actividad investigadora, a la que se suma la ejercida por diversos estudiosos de la práctica totalidad de comunidades autónomas ${ }^{16}$. Todas estas iniciativas merecerían completar el balance aquí dibujado.

Pese a que la mayoría de la actividad investigadora se desarrolla en el marco de las universidades, según venimos mencionando, no debe eludirse, por otra parte, la presencia de grupos adscritos a diferentes asociaciones autonómicas de Antropolo-

${ }^{12}$ En la Comunidad Valenciana hay varios equipos, pertenecientes a la Universidad Miguel Hernández y a la Universidad de Alicante. En Elche, la Universidad Miguel Hernández cuenta con "Cultura, Turismo y (Cooperación al) Desarrollo", y con "Economía, Cultura y Género". En Alicante, algunos antropólogos cooperan con el "Grupo Interdisciplinario de Estudios Críticos y de América Latina". Y, en Valencia, encontramos a "Inmigración, Interculturalidad y Transnacionalismo" y al "Grupo de Antropología Social" que estudia antropología de la ciencia, identidad y patrimonio, y relaciones y sistemas de género. Ciertos antropólogos de esta universidad colaboran asimismo con "Bienestar, Problemas y Políticas Sociales" y con "Ideología, Territorio y Ciudadanía".

${ }^{13}$ En esta comunidad está "Pesca, Turismo, Migraciones y Gestión de Recursos Naturales (Pescatur)", del Área de Antropología Social de la Universidad de La Laguna, donde algunos antropólogos se han integrado en "Turilab-Grupo de Investigación sobre Turismo", "Turismo, Competitividad y Sostenibilidad", y "RELICAN (Religiones en Canarias)".

${ }^{14}$ En Navarra, el equipo "Antropología Social" se ha especializado en antropología de la salud, inmigración y construcción de identidades hegemónicas dentro de los sistemas de sexo y género. Junto a él, "Cultura y Desarrollo Lera-ikergunea", basándose en la etnografía, se centra en intervención sociocomunitaria, ecosistemas de montaña y biorregiones y bienestar.

${ }^{15}$ En el País Vasco, aunque no hay constancia de colectivos estables y reconocidos como tales, debe señalarse la participación a título personal de diferentes antropólogos en proyectos vinculados a la salud y al cuerpo. De la misma manera, resulta preciso aludir tanto a la notable actividad desplegada por la asociación Ankulegi como al proyecto Etniker, llevado a cabo por el Departamento de Etnografía del Instituto Labayru, que persigue la recopilación sistemática de materiales etnográficos en el área territorial de Vasconia.

${ }^{16}$ Asumiendo que en este repaso no estarán citados todos los que son, a fin de completarlo, podemos agregar aún algunos colectivos más. En Aragón, se encuentra el "Grupo de Investigación sobre la Sociedad del Riesgo", de la Universidad de Zaragoza. En la Universidad de Extremadura, hallamos al "Grupo de Estudios Sociales Aplicados", dedicado a la frontera, la construcción sociocultural de la identidad, las relaciones interculturales, y los nexos entre patrimonio y Antropología. Para esta misma comunidad, reseñamos también a "Música e Identidad Extremeña", que ahonda en los vínculos entre Antropología, educación y música, y al "Grupo de Estudios Etno-históricos". En Murcia se localiza "Antropología y Procesos Migratorios", de la Universidad Católica de Murcia. Paralelamente, algunos antropólogos de la Universidad de las Islas Baleares cooperan con "Política, Trabajo y Sostenibilidad", orientado a procesos identitarios, migraciones y culturas del trabajo. En Castilla-La Mancha, diversos antropólogos se han adscrito a grupos de estudio de la Universidad de Castilla-La Mancha como "Investigación y Estudios de Género" o "Estudios sobre las Sociedades Árabes y Musulmanas". En Castilla y León, subrayamos las iniciativas del "Centro de Antropología Aplicada" y del "Grupo de Estudios Históricos y Antropológicos de Iberoamérica, ambos de la Universidad de Valladolid. En la Universidad de Salamanca y de León trabajan diferentes antropólogos, aunque no hay constancia de ningún grupo de investigación. Finalmente, tampoco se ha podido cotejar la existencia de equipos en Cantabria, Asturias y La Rioja, aunque en esta última comunidad el colectivo "Enclave Social", de la Universidad de La Rioja, incluye a la Antropología Social entre sus orientaciones investigadoras. 
gía cuyo grado de actividad es variable ${ }^{17}$. Del mismo modo, a estas iniciativas se unen las efectuadas en museos u otros espacios más o menos institucionalizados.

Así pues, aunque estas líneas sólo pretenden ser una aproximación al panorama investigador en Antropología Social en el Estado español, lejos de su examen exhaustivo, se puede afirmar que actualmente el balance de estudios disciplinares es muy amplio tanto por el número de grupos que los protagonizan como por la extensión y variedad de sus líneas de trabajo. Todo ello es un indicio creciente de la consolidación de la Antropología Social y de su reconocimiento social.

\section{Presentación del monográfico: temas clásicos y nuevos dominios en el es- tudio de las sociedades contemporáneas}

Comenzando por los temas contenidos en los diversos artículos de este monográfico, sorprende la mezcla de dominios clásicos de la Antropología como el parentesco, la identidad o la relación con el medio, con otros asuntos que únicamente han merecido la atención de los antropólogos en los últimos años: construcción social y cultural del amor y de los afectos, papel de los medios de comunicación en las representaciones sobre diferentes fenómenos, o el patrimonio cultural. Asimismo llama especialmente la atención la cantidad de trabajos que se centran en la migración, ya sea de forma directa o indirecta, para abordar otras temáticas. Lo muestran con claridad, de uno u otro modo, los textos de Suárez; García Castaño, Álvarez y Rubio, pero también resulta observable en los artículos de Roca y de Clua. Sin duda, tal interés obedece al incremento de la importancia que este fenómeno desempeña en la sociedad española desde las últimas décadas.

No obstante, más allá de la emergencia de este fenómeno, se puede relacionar el acercamiento de la Antropología Social al estudio de las migraciones con dos cuestiones más. Por un lado, este relieve respondería a la necesidad, cada vez mayor, de la administración estatal de conocer este hecho social. De esta manera, según apuntaba Sayad (2010), el estudio de la migración se presenta como un objeto socio-políticamente sobredeterminado, en el que una población, que ocupa posiciones subordinadas del espacio social, es sometida a una exhaustiva observación en torno a su condición de extranjeros pobres. Por otro lado, en lo relativo a la disciplina, parece posible argumentar que la Antropología Social ha descubierto en este campo analítico un lugar donde cultivar y desarrollar su producción científica, al ser el migrante un sujeto pensado y narrado como un "otro" diferente, conforme refieren en su artículo García Castaño, Álvarez y Rubio. Todo ello hace que el estudio de esta

\footnotetext{
${ }^{17}$ En el marco de las asociaciones autonómicas, hay que mencionar a los diferentes grupos de trabajo e investigación del Instituto Madrileño de Antropología (IMA) y del Instituto Catalán de Antropología (ICA). Este último centro merece una mención especial por su intensa actividad. Dispone de diversos equipos que reflexionan sobre: Religión, ritual y poder; y antropología de la locura, del cuerpo, y sonora. Cuenta, a la par, con las investigaciones llevadas a cabo por: "Ciudadanías: Antropología Histórica de las Identidades Políticas en el Estado Moderno"; "Grupo de Etnografía de los Espacios Públicos"; "Grupo de Análisis Antropológico del Arte y de la Experimentación Creativa"; "Grupo de Investigación sobre el Islam y la Diáspora"; "Periferias Urbanas"; "ERAPI (Equipo de Investigación en Antropología de los procesos Identitarios)"; y "Turiscopia”.
} 
temática se haya convertido en un área etnográfica de conocimiento, privilegiado tanto desde el punto de vista académico como político.

También en el lo relativo a los temas, quiero destacar dos características más de los artículos presentados. En primer lugar, en buena parte de ellos se advierte la creciente importancia otorgada a la forma en que el modo de producción capitalista penetra sobre los diferentes dominios culturales analizados. En este sentido, el examen de los fenómenos de mercantilización y del papel del mercado sobre los procesos sociales y culturales resulta una constante, aunque, por ejemplo, los textos de Márquez, Montesinos y Sarkis; Roca; y Herrero lo plasmen singularmente.

A la vez, algunos artículos se hacen eco de la significación actual de las nuevas tecnologías como productoras y difusoras de representaciones en ciertos enclaves determinados, lo que constituye un nuevo campo de estudio y, al mismo tiempo, un reto metodológico para la etnografía. Así lo abordan Suárez, al ocuparse de la creación de identidades y representaciones en torno a la migración producida en el espacio político-mediático; Roca, en su estudio del papel de Internet para la búsqueda virtual de pareja transnacional y de las novedades que ofrece el ciberespacio en la construcción del amor, del noviazgo y de los afectos; o Herrero, en su reconocimiento de las nuevas tecnologías como artífices de novedosas imágenes sobre el patrimonio.

Por otra parte, desde el punto de vista teórico, se aprecia en buena parte de los textos una ruptura con categorías reificadoras y esencialistas y una huida de visiones estáticas en el análisis de los contenidos de la cultura. Lo corrobora, por ejemplo, el estudio de Cantón y Gil Tébar. A esto se suma un nítido empeño por trascender y repensar bajo una perspectiva crítica ciertas dicotomías fuertemente establecidas en las ciencias sociales, en particular la oposición entre naturaleza y cultura. El trabajo de Clua es un buen ejemplo de cómo se repiensan las categorías en el ámbito de la etnicidad. De la misma forma, la noción de resiliencia, utilizada por Escalera y Ruiz, respondería en cierta medida a esta cuestión.

Otro rasgo reseñable es el reconocimiento de la etnografía en la visibilización de la heterogeneidad existente en la vida cotidiana, a lo que se asocia la significación atribuida a los grupos subalternos como sujetos activos frente a las imágenes estáticas, inmóviles y pasivas que frecuentemente se vierten sobre ellos. Lo contemplan concretamente, por ejemplo, los textos de Suárez, Cantón y Gil Tébar.

Pero, junto a estos asuntos, la característica más importante, que define a la práctica totalidad de los estudios de este monográfico, consiste en la necesidad de acudir como campo de trabajo a un marco analítico global que encuadre las interconexiones e interrelaciones entre fenómenos socioculturales geográficamente distantes entre sí. Ligado a ello, se asume la importancia de considerar la escala global en el enclave local de investigación, y las articulaciones y dialécticas entre lo local y lo global. Esto nos indica hasta qué punto está hoy totalmente superado el ideal de trabajo de campo presente en la Antropología clásica y el paradigma funcionalista, con su construcción de objetos de estudio cerrados, aislados y bien delimitados.

Desde el punto de vista metodológico, el imperativo de recurrir a una escala analítica global y de aprehender las articulaciones territoriales bajo otra óptica ha 
multiplicado la aceptación de la etnografía multisituada como estrategia óptima, que priman los trabajos aquí presentados. Además algunos de ellos, siguiendo un ideal holista, se preocupan especialmente por cartografiar, establecer y captar vínculos entre fenómenos y espacios separados geográficamente y, en apariencia, inconexos.

También en el terreno metodológico, hay que comentar la adopción en buena parte de los textos de enfoques longitudinales y perspectivas que enfatizan los elementos dinámicos. De hecho, ciertos artículos acentúan singularmente la elección de una óptica procesual para la investigación del cambio. Así ocurre, por ejemplo, en la aportación de Clua y de Cantón y Gil Tébar, al analizar la formación de categorías que resaltan respectivamente la "mezcla" étnica y la formación de los procesos de construcción identitaria. También lo expresan Escalera y Ruiz Ballesteros, aludiendo al uso de la noción de resiliencia y a sus posibilidades conceptuales, o Cátedra, cuando se refiere al carácter cambiante del patrimonio cultural.

Igualmente, resulta destacable que muchos de los artículos recurren a una perspectiva comparada como procedimiento para estudiar una misma temática. En esta línea se sitúan claramente, por ejemplo, Márquez, Montesinos y Sarkis, al analizar la ordenación de la propiedad; Escalera y Ruiz, con su presentación del concepto de resiliencia; Grau, en su examen transcultural de la noción de adopción; o Cantón y Gil Tébar, cuando se refieren a la relación entre etnicidad, religión y política.

Pasando a comentar de forma más detenida cada uno de los artículos, el monográfico comienza con el texto de Jorge Grau - "Grupo de Investigación en Antropología Fundamental y Orientada (GRAFO)"-, que se sumerge en uno de los ámbitos de investigación más clásicos de la Antropología: el parentesco. Centrándose en la adopción internacional, como fenómeno socio-jurídico, y en el sustrato y las posibles proyecciones etnocéntricas que pudieran subyacer bajo este asunto, analiza hasta qué punto dicho concepto no es una forma específica más de circulación de menores de las existentes en muchas sociedades. Tras realizar un amplio resumen de los diferentes acercamientos efectuados a la adopción internacional desde distintas disciplinas, el autor presenta algunas aportaciones realizadas desde la Antropología Social. Para ello, se basa sobre todo en el cuestionamiento que la disciplina ha realizado en torno a las fronteras biológicas del parentesco. Posteriormente, y partiendo de un enfoque comparativo y transcultural, Grau recorre el fenómeno de la circulación de menores en diferentes sociedades, para acabar interrogándose sobre las posibles elaboraciones culturales existentes en los procesos de adscripción y crianza, referidos a la adopción. Esta perspectiva comparada y transcultural, fundada en la mirada etnográfica, le permite repensar el concepto de adopción, analizarlo y definirlo como un modo más de circulación de niños, siempre que dicha noción se entienda como un conjunto de normas, prácticas y representaciones donde la responsabilidad última en el cuidado y la crianza de niños y niñas queda bien establecida. En suma, el texto de Grau es un buen ejemplo de las posibilidades de la etnografía para ver y analizar las elaboraciones culturales que la sociedad lleva a cabo sobre cuestiones como el proceso procreativo, la adscripción de los niños y las niñas, el cuidado y la crianza. Y, al mismo tiempo, nos muestra desde una pers- 
pectiva comparada la enorme variedad de formas con las que los diferentes sujetos responden a este hecho.

Montserrat Clua - “Antropología e Historia de la Construcción de Identidades Sociales y Políticas (AHICISP)"- se adentra en otro tema clásico de la Antropología: la etnicidad, argumentando que los estudios sobre ella han ido abandonando progresivamente los postulados primordialistas, singularmente a partir de las aportaciones de Barth (1976) y su enfoque situacional e interaccionista, para resaltar el carácter histórico y sociocultural que opera en la construcción de la identidad étnica. A la vez, alude a que la emergencia de nuevas identidades de índole muy variada en la sociedad contemporánea ha propiciado que las ciencias sociales presten una gran atención a este fenómeno, como sucede especialmente en los estudios poscoloniales. Partiendo del caso concreto de la emergencia del concepto de charnego para designar a la persona que vive en Cataluña procedente de otras zonas del Estado españo ${ }^{18}$, Clua se centra desde una mirada etnográfica en las dificultades que presenta la idea de mezcla en los sistemas de clasificación social, tratando de comprender las condiciones históricas y socio-culturales que envuelven la construcción $-\mathrm{o}$ no- de categorías de mestizaje y la aparición de una población mestiza como entidad social.

El texto de Clua constituye también un buen ejemplo de análisis sobre la forma en que los nacionalismos abordan e incorporan esta realidad de mezcla en los discursos identitarios. A su vez, su perspectiva procesual permite ver cómo se construyen las categorías de clasificación socio-cultural, y se adaptan y varían respecto a contextos históricos y socio-políticos específicos. Y su contenido parece sugerir, en mi opinión, un posible tema interesante de investigar desde una perspectiva procesual: los cambios y permanencias en los discursos y representaciones sobre las diferentes oleadas migratorias vividas en Catalunya, y, en particular, la migración de las pasadas décadas de 1960 y 1979, así como la acaecida en los últimos quince años, protagonizada sobre todo por población extranjera y extracomunitaria.

A continuación, el monográfico recoge el artículo de Cantón y Gil Tébar - "Transformaciones Socioculturales en Andalucía y Ameríca Latina"-, quienes, ocupándose de un campo clásico de la Antropología como es la religión, lo exploran de un modo nuevo y particular. Así, ambas autoras reflexionan sobre el papel que juega la religión como forma de resignificación del sentido de pertenencia al grupo étnico y elemento delimitador entre lo intracultural y lo intercultural. Con ello, sitúan su análisis en el triángulo establecido entre religión, etnicidad y política, mostrando el potencial de la primera en el proceso de creación de una política identitaria. Los dos estudios de caso expuestos en el artículo expresan además los procesos de innovación, transformación e hibridación que se producen en torno a lo religioso, un dominio que frecuentemente ha sido presentado como estático e inmutable.

Hay dos aspectos que son especialmente remarcables. Por un lado, el texto es un buen ejemplo de análisis de los procesos de etnogénesis y de la movilización intencional y recreación cultural que un grupo puede efectuar utilizando sus recursos

${ }^{18}$ Respecto a la temática de Clua, hay que citar la novela escrita por Francisco Candel en 1967, que tenía el sugerente título de "Els altres catalans". 
étnicos y religiosos. En segundo lugar y más importante, nos previene acertadamente sobre la representación homogeneizadora y armónica que se hace en muchas ocasiones de los "sectores marginados", a quienes en ocasiones se representa como un todo homogéneo por compartir una posición común de subalternidad. En este contexto, se resalta el papel que desempeña la etnografía como una poderosa herramienta para descubrir la pluralidad del mundo social y la heterogeneidad escondida tras la representación de las diversas formas de alteridad.

Tras los trabajos centrados en el parentesco, los sistemas de clasificación social y la identidad y la religión, el monográfico presenta dos artículos relacionados con la antropología ecológica que ahondan en la interacción establecida entre naturaleza y sociedad.

El interesante estudio de Escalera y Ruiz Ballesteros - "Grupo de Investigación Social y Acción Participativa (GISAP)" - constata el progresivo acercamiento entre los enfoques biofísicos y los socioculturales, así como la necesidad de tender a una construcción transdisciplinar que integre ambos enfoques y reconozca la intensa interrelación existente entre la dimensión sociocultural y la ecológica. En la búsqueda de esa interrelación, se señala cómo la sensibilidad ambiental es cada vez más socioambiental, dada la multiplicación de las investigaciones que subrayan la importancia de las variables socioculturales en la relación con el medio. En coherencia con este planteamiento y tras la huella de un enfoque integrador de esas dos dimensiones, los autores aluden a dos nociones. En primer lugar, con el concepto de socioecosistema se persigue incorporar las relaciones sociales al ecosistema y explorar los vínculos de éstas con él. De hecho, se asume que ambos configuran un "sistema adaptativo complejo". Al mismo tiempo, esta noción busca diluir la dicotomía naturaleza-cultura incorporando al análisis aspectos como las relaciones de poder o la autonomía de los grupos humanos. En segundo término, la noción de resiliencia nos permite acercarnos a la capacidad de un socioecosistema para afrontar el cambio sin alterar sustancialmente su forma y funciones, en una especie de "conservación creativa". De este modo, con dicho concepto se trata de presentar la relación del grupo con el medio de una manera dinámica, que supere las nociones tradicionales de equilibrio tan presentes en las ciencias sociales. El objetivo es enfatizar los aspectos procesuales y de cambio para procurar integrar la inestabilidad, la complejidad o la incertidumbre existentes en la realidad. En este contexto, los dos estudios de caso presentados aportan múltiples claves sobre cómo hacer operativo en el análisis ese concepto, e igualmente acerca de los aspectos sociales más relevantes a los que debe prestarse atención para definir un enfoque de estas características y proceder con él. Asimismo, los casos contemplados en este artículo son un buen ejemplo de la significación que la etnografía, como método, y la antropología, como disciplina, pueden protagonizar en la adopción de una perspectiva transdisciplinar holística e integradora, que se proponga desbordar los límites tradicionales del conocimiento entre las ciencias naturales y sociales.

Al igual que en el texto de Escalera y Ruiz, Elías Zamora y Rufino Acosta - "Cultura, Ecología y Desarrollo de Pequeños Territorios"- fijan el núcleo de su reflexión en la relación entre ecosistema y población. Sin embargo, la novedad de 
este artículo respecto al anterior estriba en ahondar, bajo una perspectiva analítica estructural, en las líneas de fractura y división que caracterizan a los sujetos sociales al usar sus recursos. De este modo, el análisis de la posición de poder ocupa un lugar preeminente sobre la gestión de los recursos, una cuestión que aparece de forma más difusa en el estudio anterior. A partir del análisis discursivo, el texto evidencia la diferente posición respecto al uso del agua que tienen diversos sectores de la población en función de su relación con la utilización de los recursos hídricos y su empleo en la producción agrícola. Se muestran también las distintas perspectivas y utilizaciones que se hacen de los discursos "técnicos" y científicos desde las dispares posturas enfrentadas. A la par, el caso examinado es un buen ejemplo de la influencia e interdependencia ascendente que tiene el marco global sobre el local, conforme sucede con las demandas sobre la gestión hídrica procedentes de otras instituciones como la UE y su Directiva Marco del Agua. Finalmente, debe destacarse la dimensión aplicada de este trabajo, dado que, a través del análisis realizado sobre las diferentes lógicas y discursos subyacentes al uso del agua, se pretende ofrecer elementos que faciliten la gobernanza y la toma de decisiones visibilizando los divergentes "puntos de vista" expresados por los diversos actores sobre un mismo tema.

Márquez, Montesinos y Sarkis — "Grupo de Estudios sobre Reciprocidad"analizan en su artículo las transformaciones producidas en el régimen de propiedad, aportando tres estudios de caso comparados: la ordenación de un asentamiento informal de viviendas, la redefinición de las relaciones de aparcería y la organización del uso comunal de la tierra. Un elemento común atraviesa estas tres experiencias: la penetración inducida por el ordenamiento jurídico de una lógica de individualización en las relaciones de propiedad que, bajo el argumento de otorgar mayor seguridad a los poseedores, conlleva un reforzamiento del vínculo entre el ciudadano y el Estado. Se alude a cómo este proceso fomenta el debilitamiento del consenso del grupo, la introducción de una lógica utilitarista y economicista que prima los intereses del mercado, y el afianzamiento del control del Estado sobre el territorio y las relaciones económicas. Dos cuestiones relevantes se despenderían de ello. La primera es que la etnografía permite mostrar una perspectiva microsocial de la praxis legal, al visibilizar las consecuencias de la plasmación de este proceso de ordenamiento jurídico, así como las relaciones y diferencias en las lógicas existentes entre las leyes oficiales y consuetudinarias. La segunda es la importante contribución de esta reflexión al interesante debate sobre la economía moral, planteado hace años por Thompson (1991), al estudiar las concepciones morales, inherentes a las diferentes prácticas promovidas, y fundamentar los posibles conflictos que pueden desencadenarse entre los regímenes consuetudinarios y aquellos promovidos por el aparato estatal.

A continuación, encontramos varios textos cuyo denominador común es la temática migratoria. Estas aportaciones tratan aspectos muy diferentes que, sin embargo, las complementan entre sí, al tiempo que nos dotan de una perspectiva óptima para valorar la investigación sobre la etnicidad desarrollada por Clua. En estos artículos el análisis de la migración se integra en el amplio marco del conjunto de las 
relaciones sociales, incluyéndose en todos ellos asuntos novedosos vinculados con dicho campo de estudio. Estos trabajos se caracterizan además por querer construir un proyecto antropológico, epistemológicamente reflexivo, que trascienda en sus análisis ciertas prenociones del fenómeno migratorio, firmemente arraigadas en el sentido común hegemónico.

El primero de estos textos es el de García Castaño, Álvarez Veinguer y Rubio Gómez - "Laboratorio de Estudios Interculturales (LdEI)"-, donde se recogen algunas de las contribuciones efectuadas por la Antropología Social a los estudios migratorios y se reflexiona sobre la etnografía multisituada como propuesta metodológica cada vez más aceptada. Este artículo es, a la par, un buen exponente de las introspecciones metodológicas llevadas a cabo en un contexto de análisis, caracterizado por la necesidad de atender la articulación entre la realidad local y el sistema mundial, así como la ascendente interconexión e interdependencia cuantitativa y cualitativa que existe entre las distintas regiones del mundo. Varias son las razones aludidas para argumentar la especial notoriedad de la etnografía multisituada, como estrategia metodológica, en la investigación de las migraciones internacionales. En primer lugar, ello se debe a la trascendencia en estos estudios de la perspectiva transnacional, un enfoque que prima en su análisis los vínculos establecidos por los migrantes entre su lugar de origen y destino, y que ve en los postulados de la etnografía una herramienta metodológica privilegiada (Levitt y Glick Schiller, 2004). En segundo término, responde al relieve de las aportaciones de autores como Sayad (2010), quien destaca la importancia de acudir al contexto de partida de los migrantes en la investigación de sus procesos de movilidad. Pero, más allá de las disquisiciones emprendidas respecto al papel de la etnografía en el contexto global, quizás la contribución más novedosa de este texto es su reflexión sobre la etnografía multisituada como una creación de prismas transescalares. Esta metáfora nos invita a mirar la etnografía como la construcción de un marco explicativo de un mismo fenómeno narrado desde diversos ángulos. Desde esta perspectiva, a la vez que se establecen articulaciones entre los diferentes espacios de observación dentro de un marco global, se presenta de forma interrelacionada los dispares discursos, narraciones y significados vertidos sobre el mismo fenómeno por los distintos actores sociales.

Liliana Suárez - “Grupo de Investigación Migraciones, Etnicidad y Ciudadanía"- explora en su artículo una cuestión novedosa: las representaciones vertidas sobre la población inmigrante en los medios de comunicación y la construcción en el espacio político-mediático de nuevas identidades. En la primera parte del texto, al aludir a los medios generalistas, se presenta el papel que juega el campo periodístico en la creación de las relaciones socioeconómicas y simbólicas, y su significación en la construcción de un imaginario homogeneizador de la migración, dominado por la imagen de los inmigrantes como "personas pobres". Frente a esos medios generalistas, otros especializados, desarrollados activamente en el país por y para inmigrantes, parecen haber resignificado esa imagen, traduciéndola en términos de audiencia potencial. Los motivos de esas distintas representaciones y de la propia creación y reproducción de unos y otros medios atraviesan este análisis. Y en la 
segunda parte del artículo se examina las características de los medios dirigidos hacia latinoamericanos y las razones que confluyen en la construcción de lo "latino" como una audiencia potencial homogénea y segmentada. En este sentido, resulta especialmente interesante la lectura de la categoría "latina" como una identidad emergente que, siguiendo una lógica extraterritorial, contiene un cierto carácter poscolonial y ofrece una dimensión empoderadora. Podría sostenerse que un incipiente proceso de etnogénesis gravita en torno a esa identidad "latina", favorecida desde los medios de comunicación, que remite como denominador común a la migración y una experiencia histórica compartida, y que nos permite reflexionar sobre la enorme importancia de los flujos de información y los contenidos mediáticos en la construcción de identidades emergentes.

Con una temática totalmente diferente, Jordi Roca — “Grup d'Antropología Social" - también valora la trascendencia de los contenidos mediáticos en la construcción de nuevas representaciones sobre la alteridad, a las que asimismo contribuye la extensión de las TIC. Su estudio de los noviazgos transnacionales y las búsquedas virtuales de parejas no sólo aborda un asunto etnográficamente muy novedoso, sino que nos induce a reflexionar sobre los "cambios surgidos en el territorio amoroso". Localizado en la intersección entre la perspectiva transnacional y los estudios sobre flujos transnacionales globalizados, parentesco, emociones y mercantilización de la intimidad, este trabajo acude a la etnografía multisituada con el fin de analizar los vínculos existentes entre las experiencias vitales y los fenómenos infra y supraestructurales que posibilitan la aparición del fenómeno investigado. Así, se hace un original recorrido por el papel del espacio virtual y de los portales y agencias especializados en citas, presentando estos espacios como lugares para la mercantilización de un individuo, exhibido como un producto para su tasación, compra-venta o descarte. Respecto a las redes románticas y agencias matrimoniales, destacaría dos cuestiones. En primer lugar, son llamativas las semejanzas y diferencias entre las relaciones establecidas en el espacio virtual y el real, dado que en este último ámbito las estrategias de presentación del yo y el acercamiento deben adaptarse a la ausencia de señales del contexto social y espacial. En segundo término, resulta muy sugerente la reflexión efectuada de la tensión narrativa, expresada en muchos testimonios, entre el intento de presentar la relación como algo alejado de la lógica mercantil y la construcción de un discurso más en sintonía con el amor romántico normativo. Finalmente, en un contexto donde las relaciones sociales cada vez están más mediatizadas tecnológicamente, este artículo nos aporta elementos para pensar las formas de articulación entre el espacio real y virtual. Rompe también la imagen de superficialidad que, en muchas ocasiones, se vierte en las aproximaciones a lo virtual. Todo ello, nos lleva a enfatizar el relieve que puede tener la etnografía en los análisis sobre el uso e impacto de las TIC, el campo virtual, o la articulación entre éste y lo real; unos ámbitos de estudio sumamente atractivos, pero que no están exentos de retos y desafíos metodológicos.

El monográfico se cierra con dos artículos cuyo eje principal es el patrimonio cultural. El primero de ellos es el de Nieves Herrero - "Antropoloxia"-, quien se aproxima a los procesos de patrimonialización de la cultura en el marco de la 
posmodernidad, a la que reconoce capaz de extender las formas culturales y la hegemonía de la imagen al conjunto del ámbito social y de anular la distinción entre lo real y lo imaginario e incluso el propio sentido de la historia. Herrero explora la denominada "segunda ola" de patrimonialización de la cultura, que viene a reproducir e incrementar el interés surgido a partir de la vinculación que con anterioridad se promovía entre patrimonio e identidad nacional. En contraste con situaciones previas, esta "segunda ola" de patrimonialización de objetos, prácticas y dominios culturales está presidida por la búsqueda de recursos susceptibles de generar riqueza económica, principalmente asociada con el turismo, y sigue la lógica de los mercados y sus reclamos a través de los medios masivos de comunicación. Finalmente, basándose en su investigación del Camino de Santiago y con el objetivo de aplicar sus conclusiones a una eventual intervención en la gestión del patrimonio, la autora resalta diversos aspectos que resultan imprescindibles y dignos de ser potenciados: la creación en torno a éste de capital social e identitario, la importancia del espacio local o el impulso a la educación patrimonial.

Por último, María Cátedra - “Antropología de las Políticas Sociales y Culturales (APSYC)"-, centrándose en las sucesivas restauraciones llevadas a cabo en el Templo de Diana de Évora, nos ofrece un texto donde la historia dialoga con diversas nociones que han ido surgiendo en torno al patrimonio, cuya conceptualización, lejos de ser neutra, expresa una forma histórica, socialmente construida y cambiante. Así, en su amplio repaso de las consideraciones y posicionamientos, que se han realizado sobre este templo en diferentes épocas, se puede observar la distinta y cambiante percepción que la sociedad ha ido teniendo de su historia y de su patrimonio. De este modo, una lectura diacrónica de ese monumento permite visualizar los diversos sentidos y aspectos que se han ido enfatizando y poniendo en valor a lo largo de la historia: el carácter conmemorativo del monumento, su importancia como vestigio de la antigüedad, su valor económico... Asimismo se resalta el papel de los monumentos, e incluso, de las ruinas como elementos privilegiados para interpretar la ciudad y el territorio local, e igualmente la forma en la que los habitantes crean sentido en torno al espacio público habitado y a la experiencia social compartida.

\section{4. Últimos comentarios}

Como puede concluirse de los textos recogidos en este monográfico y de las líneas de investigación de distintos equipos, la Antropología Social ha ido ampliando progresivamente sus ámbitos de estudio hasta acercarse a un conjunto de temáticas enormemente variadas, donde se conjugan dominios clásicos de trabajo con otras líneas de trabajo más recientes.

Si nos acercamos a las líneas de investigación de los diferentes equipos y a los textos aquí presentados, podemos apreciar que, a pesar de unas realidades muy cambiantes, la etnografía sigue teniendo una importancia central como herramienta que permite arrojar luz sobre aspectos no contemplados por otras disciplinas u otras aproximaciones metodológicas. La etnografía, cuyas señas de identidad están basadas en la perspectiva microanalítica y la recogida de datos sobre el terreno (Agier, 
1996), es el instrumento metodológico para "rescatar una mirada 'de cerca de dentro' capaz de identificar, describir y reflejar aspectos excluidos de la perspectiva de aquellos enfoques que, por efecto de contraste, calificamos como de fuera y de lejos"19 (Magnani, 2002: 11).

Sin embargo, la Antropología como disciplina no está exenta de desafíos; y más en el marco de la sociedad postindustrial y en un contexto caracterizado por la creciente aceptación de lo global como escala analítica, la necesidad de reconocer las interacciones operadas entre diferentes lugares territorialmente separados entre sí, y el reto de abordar las articulaciones que se producen entre la realidad local y el sistema mundial. Algunos de estos desafíos, ya fueron señalados por Cruces (2003): la necesidad de repensar la pretensión holística de la disciplina en el nuevo contexto ante las dificultades para efectuar un mapeo totalizador; los cambios en la cronotopia tardomoderna y el contraste entre ésta y el relato etnográfico clásico; o la incomensurabilidad de saberes que se produce al no existir una separación clara en el nuevo contexto entre observador y observado.

No obstante, pese a los desafíos presentes, comparto la afirmación de Cruces, cuando menciona que la etnografía sigue constituyendo la aportación más viva que ha brindado la Antropología al desarrollo del diálogo en las ciencias sociales. Queda por ver qué nuevas formas de imaginación teórico-metodológica se acometerán en el futuro para abordar esos retos y, sobre todo, cómo se puede hacer — parafraseando a Gabriel Celaya - para que la Antropología continúe siendo un arma cargada de futuro.

\section{Referencias bibliográficas}

AGIER, Michel

1996 "Les savoirs urbans de l'anthropologie". Enquête, 4 : 35-58.

BARTH, Frederik

1976 Los grupos étnicos y sus fronteras. México: Fondo de Cultura Económica.

CRUCES, Francisco

2003 "Etnografías sin final feliz. Sobre las condiciones de posibilidad del trabajo de campo urbano en contextos globalizados". Revista de Dialectología y Tradiciones Populares, LVIII, 2: 161-178.

LEVITT, Peggy; GLICK SCHILLER, Nina

2004 "Perspectivas internacionales sobre migración: conceptualizar la simultaneidad". Migración y Desarrrollo, Segundo Semestre, 60-91. Disponible en: www.migracionydesarrollo.org.

${ }^{19}$ Es traducción del autor. 
MAGNANI, José Guilherme Cantor

2002 "De perto e de dentro: notas para uma etnografia urbana". Revista Brasileira de Ciencias Sociais, 17, 49: 11-29. Disponible en: http://redalyc.uaemex.mx/redalyc/ src/inicio/ArtPdfRed.jsp?iCve=10704902

MARCUS, George E.

1995 "Ethnography in/of the World System: The Emergente of Multi-sited Ethnography". Annual Anthropological Review, 24: 96-117.

PRAT, Joan (Coord.)

1999 Investigadores e investigados: Literatura antropológica en España desde 1954. Tarragona: Arxiu d'Etnografía de Catalunya, Tarragona, Institut Tarragonés d'Antropología, Federación de Asociaciones de Antropología del Estado Español.

SAYAD, Abdelmalek

2010 "Una sociología (de las migraciones) para la resistencia". Empiria: revista de metodología de ciencias sociales, 19: 235-276.

THOMPSON, Edward P.

1991 "The Moral Economy Reviewed", en Customs in Common. New York: New Press, 259-351. 